\title{
Decreased Plasma Nesfatin-1 Level Is Related to the Thyroid Dysfunction in Patients with Type 2 Diabetes Mellitus
}

\author{
Fupeng Liu, ${ }^{1}$ Qing Yang, ${ }^{2}$ Ning Gao, ${ }^{3}$ Fangfang Liu, ${ }^{3}$ and Shaohua Chen ${ }^{3}$ \\ ${ }^{1}$ Department of Endocrinology, Central People's Hospital of Tengzhou City, Tengzhou, Shandong 277500, China \\ ${ }^{2}$ Department of Medicine, Maternal and Child Care Service Centre of Tengzhou City, Tengzhou, Shandong 277500, China \\ ${ }^{3}$ Department of Endocrinology, Qianfoshan Hospital of Shandong University, Jinan, Shandong 250000, China
}

Correspondence should be addressed to Shaohua Chen; chenshaohua@medmail.com.cn

Received 27 March 2014; Accepted 21 May 2014; Published 4 June 2014

Academic Editor: Hiroshi Okamoto

Copyright (C) 2014 Fupeng Liu et al. This is an open access article distributed under the Creative Commons Attribution License, which permits unrestricted use, distribution, and reproduction in any medium, provided the original work is properly cited.

\begin{abstract}
Aims. Thyroid dysfunction is frequently observed in patients with type 2 diabetes mellitus (T2DM), but the underlying mechanism is still poorly understood. The present study aimed to investigate whether nesfatin-1 played a role in the thyroid dysfunction in patients with T2DM. Methods. 55 euthyroid patients were enrolled in this study including 30 patients with T2DM and 25 patients with impaired glucose regulation (IGR). 30 age-matched healthy people were also included as the control. The plasma levels of nesfatin-1, thyrotropin (TSH), and glycosylated hemoglobin (HbAlc) as well as the body mass index (BMI) were comparatively analyzed among the three groups. Results. The nesfatin-1 was significantly lower in patients with T2DM than in patients with IGR and in the control. On the contrary, the TSH level was significantly higher in patients with T2DM than in patients with IGR and in the control. Simple regression analysis showed that the plasma nesfatin-1 was negatively correlated with the TSH and HbAlc levels and positively correlated with the BMI. With multiple stepwise regression analysis, the nesfatin-1 remained to be independently correlated with the TSH, BMI, and HbAlc. Conclusions. The study was suggesting a role of nesfatin-1 in thyroid dysfunction in patients with T2DM.
\end{abstract}

\section{Introduction}

Thyroid dysfunction is frequently observed in patients with type 2 diabetes mellitus (T2DM), but the underlying mechanism is still poorly understood [1-3]. Nesfatin1 is a peptide that has been identified in the hypothalamus including the paraventricular nucleus (PVN) and is encoded in the $\mathrm{N}$-terminal region of the precursor protein NEFA/nucleobindin-2 (NUCB2) [4]. A major function of the nesfatin-1 is inhibition of food intake and regulation of blood glucose in time-, dose-, and insulin-dependent manners [4-7]. Studies have shown that nesfatin-1 is colocalized with thyrotropin-releasing hormone (TRH) and affects the membrane potential of TRH neurons in the PVN, which is known to be closely related to the regulation of thyroid function [8-10]. Also interesting, Sawicka et al. have reported that the unusual thyroid hormone level is associated with the change of ghrelin expression, while ghrelin has been shown to be coexpressed with nesfatin-1 in gastric X/A-like endocrine cells $[11,12]$. These findings indicate that nesfatin-1 may have a close relationship with thyroid function. In the present study, we explored the relationship between nesfatin-1 and thyroid dysfunction in patients with T2DM by comparing the plasma levels of nesfatin- 1 and TSH between the patients with T2DM and those with impaired glucose regulation (IGR) but without thyroid diseases. Healthy people with normal glucose tolerance were used as the control. Our results suggested that the abnormal plasma level of nesfatin-1 was associated with the dysfunction of thyroid in patients with T2DM.

\section{Materials and Methods}

2.1. Patients. A total of 55 patients with abnormal glucose metabolism were enrolled in this study, including 30 patients with T2DM and 25 patients with IGR. All patients were randomly selected from those who visited the Outpatient Department of Qianfoshan Hospital, Shandong University, 
between February and September 2012. T2DM and IGR were diagnosed with the $75 \mathrm{~g}$ oral glucose tolerance test according to the definition given by World Health Organization in 1999. The IGR patients were all newly diagnosed and did not receive any diabetes medications or diets before. The mean age and body mass index (BMI) of the T2DM patients were $62.23 \pm$ 8.11 years old and $25.79 \pm 2.64 \mathrm{~kg} / \mathrm{m}^{2}$, respectively. The mean age and BMI of the IGR patients were $60.04 \pm 9.60$ years old and $25.76 \pm 2.86 \mathrm{~kg} / \mathrm{m}^{2}$, respectively. There were no significant differences for the age and BMI between T2DM and IGR patients. 30 age-matched healthy people (age, $59.13 \pm 9.45$ years; BMI, $23.71 \pm 2.65 \mathrm{~kg} / \mathrm{m}^{2}$ ) who visited our hospital for regular physical examination were selected as the controls. All the control people had a normal glucose tolerance, had no family history of diabetes or other endocrine disorders, and were not taking any medications known to change glucose tolerance. Clinical exclusion criteria included type 1 diabetes mellitus (T1DM), history of thyroid and other endocrine disorders, taking any drugs known to alter the thyroid function (e.g., antihyperthyroidism drugs, thyroxine, etc.) in the past two months, acute diabetes complications, severe infectious disease, pregnancy, surgery or trauma in recent times, heart failure, hypertension, liver or kidney disease, and cancer. All people in this study were Han Chinese. The present study was conducted based on the principles of the Declaration of Helsinki and approved by the Ethics Committee of Shandong University. Informed consent was obtained from all people in this study.

2.2. Clinical and Laboratory Examination. Physical examination of the patients including their height, weight, and blood pressure was performed in the morning before breakfast. The BMI was calculated with the formula of "BMI = weight $(\mathrm{kg}) /(\text { height }(\mathrm{m}))^{2}$." Fasting blood samples (12 h overnight fast) were collected at the same time for measurements of fasting blood glucose (FBG), fasting insulin (FINS), glycosylated hemoglobin (HbAlc), triglycerides (TG), total cholesterol (TC), high density lipoprotein cholesterol (HDL-C), low density lipoprotein cholesterol (LDL-C), free triiodothyronine (FT3), free thyroxine (FT4), thyrotropin (TSH), thyroid peroxidase antibody (TPOAb), and thyroglobulin antibody (TGAb). The fasting plasma level of nesfatin1 was measured with the enzyme-linked immunosorbent (EIA) assay kit according to the manufacturer's instruction (Phoenix Pharmaceuticals, Belmont, CA). The EIA kit had high specificity to nesfatin-1 and no cross-reactivity with ghrelin, angiotensin, visfatin, and NUCB2. The sensitivity of this assay was $0.78 \mathrm{ng} / \mathrm{mL}$ and the intra- and interassay coefficients of variation were $10 \%$ and $15 \%$, respectively. The homeostasis model assessment of insulin resistance (HOMAIR) was calculated according to the equation: HOMA-IR = fasting insulin $(\mu \mathrm{U} / \mathrm{mL}) \times$ fasting glucose $(\mathrm{mmol} / \mathrm{L}) / 22.5$.

2.3. Oral Glucose Tolerance Test. Venous blood samples from each participant were collected at $0,60,120$, and $180 \mathrm{~min}$, respectively, after glucose load. The results were interpreted according to the definition of World Health Organization Expert Committee on Diabetes Mellitus in 1999: FBG <
$6.1 \mathrm{mmol} / \mathrm{L}$ and $2 \mathrm{~h}$ postprandial blood glucose $(2 \mathrm{hPG})<$ $7.8 \mathrm{mmol} / \mathrm{L}$ for normal glucose tolerance; FBG $>7.0 \mathrm{mmol} / \mathrm{L}$ and $2 \mathrm{hPG}>11.1 \mathrm{mmol} / \mathrm{L}$ for T2DM; $2 \mathrm{hPG}<7.8 \mathrm{mmol} / \mathrm{L}, \mathrm{FBG}$ $>6.1$ but $<7.0 \mathrm{mmol} / \mathrm{L}$ for impaired fasting blood glucose; and $\mathrm{FBG}<6.1 \mathrm{mmol} / \mathrm{L}$ and $2 \mathrm{hPG}>7.8 \mathrm{mmol} / \mathrm{L}$ but $<11.1 \mathrm{mmol} / \mathrm{L}$ for impaired glucose tolerance.

2.4. Statistical Analysis. The statistical analyses were performed using the SPSS11.0 software (SPSS Inc., Chicago, IL), and the $P$ value of less than 0.05 (two-tailed) was considered to be statistically significant. All data were expressed as the mean \pm SD. One-way ANOVA with post hoc (least significant difference) analysis was used to compare mean values among the T2DM patients, IGR patients, and normal controls. Simple and multiple linear regression analyses were used to examine the relationships between the levels of fasting plasma nesfatin-1, thyroid related hormones, and other variables.

\section{Results}

Clinical characteristics and laboratory findings of the three groups were shown in Table 1. The fasting plasma level of TSH was significantly higher in the T2DM group than in the IGR and control groups $(2.10 \pm 1.09$ versus $1.50 \pm 0.73$ versus $1.46 \pm 0.60 \mu \mathrm{IU} / \mathrm{mL}$, both $P<0.05)$. There were no significant differences for other thyroid hormones and antibodies between the T2DM and other two groups. In contrast to TSH, the plasma nesfatin-1 level in the T2DM group was significantly lower than in the groups of IGR and controls $(0.73 \pm 0.14$ versus $1.12 \pm 0.39$ versus $1.00 \pm$ $0.23 \mathrm{ng} / \mathrm{mL}$, both $P<0.01$ ) (Figure 1). No significant difference was obtained for the nesfatin-1 level between the IGR and control groups (Figure 1). Patients with T2DM and patients with IGR had an increased level of BMI, FBG, FINS, and HOMA-IR, compared with the control group (all, $P<$ 0.05). The levels of FBG, HOMA-IR, HbAlc, TG, and LDL$C$ were all significantly higher in the T2DM than in the IGR patients (all, $P<0.05$ ). Varieties of FT3, FT4, TPOAb, and TGAb remained consistent among the three groups.

Simple regression analysis with the pooled data showed that the plasma nesfatin-1 level was positively correlated with the BMI $(r=0.397)$ and negatively correlated with the TSH and HbAlc levels ( $r=-0.404$ and -0.389 , resp.). The nesfatin-1 level remained independently correlated with the TSH, BMI, and HbAlc levels with multiple stepwise regression analysis (Figure 2). The multiple regression equation was as follows: $Y=-0.100 X_{\mathrm{TSH}}+0.049 X_{\mathrm{BMI}}-0.087 X_{\mathrm{HbAlc}}+$ 0.405 .

\section{Discussion}

In the present study, we found that the plasma TSH level was significantly higher, whereas the plasma nesfatin-1 level was remarkably lower in patients with T2DM, compared with the patients with IGR and the control group. With simple and multiple stepwise regression analyses, the plasma nesfatin-1 level was independently correlated with the TSH level. These 
TABLE 1: Clinical characteristics of the patients $(\bar{x} \pm s)$.

\begin{tabular}{lccc}
\hline Group & T2DM & IGR & NGT \\
\hline Age (year) & $61.23 \pm 8.83$ & $59.04 \pm 9.01$ & $59.60 \pm 9.26$ \\
Number (M/F) & $30(16 / 14)$ & $25(13 / 12)$ & $30(14 / 16)$ \\
Body mass index & $26.11 \pm 2.55^{*}$ & $26.12 \pm 2.07^{*}$ & - \\
Duration of diabetes (year) & $9.03 \pm 3.48$ & - & $24.72 \pm 2.48$ \\
Fasting blood glucose (mmol/L) & $7.37 \pm 1.15^{* * \boldsymbol{\Delta}}$ & $5.75 \pm 0.54^{* *}$ & $5.00 \pm 0.46$ \\
Fasting insulin (mIU/L) & $7.72 \pm 0.86^{* *}$ & $1.93 \pm 3.73^{* *}$ & $5.56 \pm 1.27$ \\
HOMA-IR & $2.54 \pm 0.55^{* * \boldsymbol{\Delta}}$ & $5.64 \pm 0.42$ & $1.24 \pm 0.33$ \\
HbAlc (\%) & $7.23 \pm 1.27^{* * \boldsymbol{\Delta}}$ & $5.63 \pm 0.61$ \\
Triglycerides (mmol/L) & $2.78 \pm 1.71^{* * \Delta}$ & $1.79 \pm 1.24$ & $1.57 \pm 1.09$ \\
Total cholesterol (mmol/L) & $5.56 \pm 1.14$ & $5.56 \pm 1.09$ & $5.22 \pm 0.99$ \\
High density lipoprotein cholesterol (mmol/L) & $1.43 \pm 0.29$ & $1.43 \pm 0.26$ & $1.35 \pm 0.37$ \\
Low density lipoprotein cholesterol (mmol/L) & $3.21 \pm 0.90^{\triangle}$ & $3.78 \pm 0.78^{*}$ & $3.08 \pm 1.28$ \\
Free triiodothyronine (pmol/L) & $4.72 \pm 0.63$ & $5.04 \pm 1.05$ & $5.07 \pm 0.74$ \\
Free thyroxine (pmol/L) & $16.87 \pm 2.45$ & $16.85 \pm 2.35$ & $17.21 \pm 1.58$ \\
Thyrotropin (uIU/mL) & $2.10 \pm 1.09^{* * \Delta}$ & $1.50 \pm 0.73$ & $1.46 \pm 0.60$ \\
Thyroid peroxidase antibody (pmol/L) & $1.93 \pm 1.67$ & $1.65 \pm 1.71$ & $1.59 \pm 1.67$ \\
Thyroglobulin antibody (pmol/L) & $1.64 \pm 0.78$ & $1.50 \pm 1.12$ & $1.81 \pm 2.44$ \\
\hline
\end{tabular}

Data are expressed as mean \pm SD. T2DM: type 2 diabetes mellitus; IGR: impaired glucose regulation; NGT: normal glucose tolerance; HOMA-IR: HOMAinsulin resistance index; HbAlc: glycosylated hemoglobin; $*<0.05, * *<0.01$ versus NGT group; $\Delta<0.05, \boldsymbol{\Lambda}<0.01$ versus IGR group.

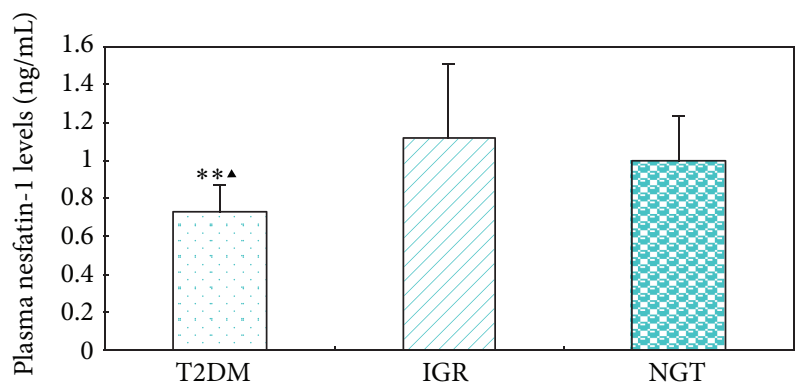

Figure 1: The plasma nesfatin-1 levels in patients with type 2 diabetes mellitus (T2DM) and patients with impaired glucose regulation (IGR) as well as in the control people with normal glucose tolerance (NGT). T2DM versus IGR group, $\boldsymbol{\Delta}<0.01$; T2DM versus NGT group, $* *<0.01$.

results suggested that decreased nesfatin-1 level might play a role in the thyroid dysfunction in T2DM patients.

Thyroid dysfunction is a common endocrine disorder, frequently induced by autoimmune processes, while the patients with diabetes often exhibit thyroid dysfunction. Studies by Radaideh et al. have shown that the overall prevalence of thyroid dysfunction is up to $12.5 \%$ in T2DM patients, in contrast to $6.6 \%$ in healthy population [13]. The most common disease of thyroid is the subclinical hypothyroidism. Sowiński et al. have demonstrated that about $15 \%$ of T2DM patients suffer from overt hypothyroidism, and further $10 \%$ present subclinical hypothyroidism [14]. However, the underlying mechanism for the thyroid dysfunction in T2DM patients remains poorly understood, although T1DM and thyroid dysfunction are known to share the similar genotypic milieu [15]. A potential mechanism might be the complex interaction of signaling pathways associated with

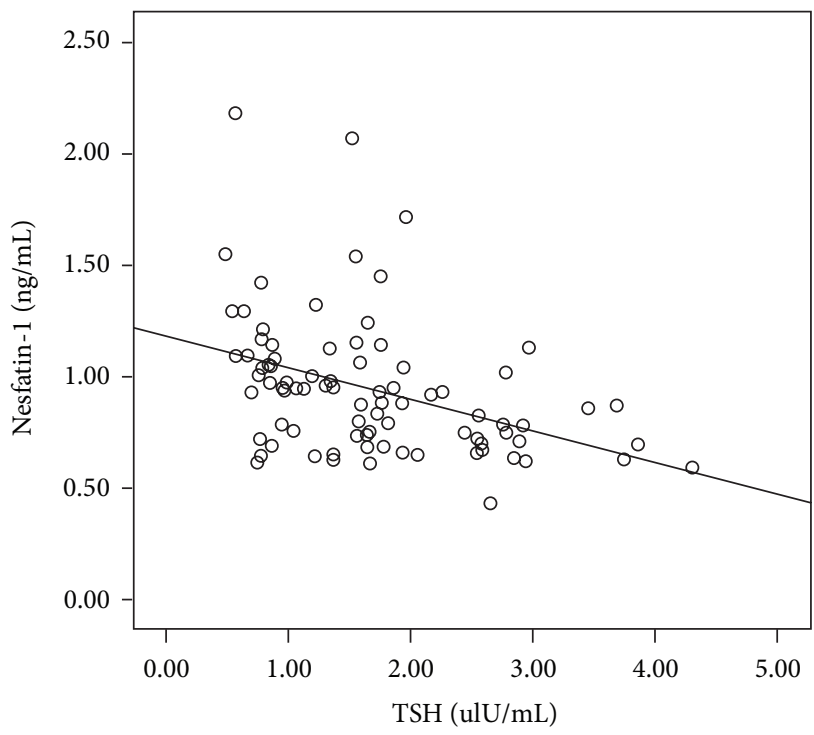

FIGURE 2: Negative correlation of the plasma nesfatin-1 level with the plasma TSH level $(r=-0.404, P<0.01)$.

the glycometabolism and energy metabolism, and the $5^{\prime}$ adenosine monophosphate-activated protein kinase (AMPK) might be a crucial target, which not only participated in the modulation of insulin sensitivity but also is involved in the feedback of thyroid hormones on appetite and energy expenditure [16-18].

Nesfatin-1 is an adipocytokine. In addition to its effect on metabolic regulation and food intake, animal studies have also suggested that nesfatin-1 could enhance insulin release and lead to a time-, dose-, and insulin-dependent reduction of blood glucose $[6,19,20]$. Yang et al. have shown that the 
central nesfatin-1 can increase the insulin receptor/insulin receptor substrate-1/AMPK/Akt/target of rapamycin complex 2 phosphorylation, resulting in an increase in Fos immunoreactivity in the hypothalamic nuclei [21]. These results suggest that nesfatin-1 may take part in the regulation of both glycometabolism and thyroid hormone functions. Also, the nesfatin-1 immunopositive neurons have been reported to be colocalized with TRH neurons in the PVN and the central nesfatin-1 affects the membrane potential of TRH neurons $[8,22]$. Thyroid hormones are tightly regulated by the hypothalamus-pituitary-thyroid axis, and a major component of this control system is the hypothalamic PVN, which contains neurons that produce TRH to regulate the TSH secretion in the anterior pituitary [9]. In the present studies, the plasma nesfatin-1 level was found to be negatively and independently correlated with the TSH level in patients with T2DM. Considering the crucial role of nesfatin-1 in energy and glucose metabolism, we speculated that it might act as a regulatory factor of thyroid function by adjusting AMPK activity and TRH secretion in T2DM patients, thereby resulting in the subclinical hypothyroidism. For the limitation of patients enrolled, we could not analyze whether decreased plasma nesfatin-1 level is related to hyper- or hypothyroidism. It is worthy of a further research.

Our finding of low plasma nesfatin-1 level in T2DM patients was consistent with other studies showing that circulating nesfatin-1 level is dramatically reduced in T2DM patients [23]. We did not obtain a difference for the nesfatin1 level between IGR and control groups, even though it was slightly increased in the IGR group. This finding was different from the result obtained by Zhang et al. showing that the nesfatin-1 concentration is elevated significantly in patients either with IGT or with newly diagnosed T2DM [24]. Patients' selection may be a possible reason for this discrepancy. Our studies further showed that the plasma nesfatin-1 level was positively correlated with the BMI and negatively correlated with the HbAlc level independently. The present study supports that nesfatin-1 may play a role in the thyroid dysfunction in patients with T2DM.

\section{Conflict of Interests}

None of the authors has any potential conflict of interests associated with this research.

\section{Acknowledgments}

The authors would like to thank the staff members in the Department of Endocrinology, Qianfoshan Hospital, for collection of patients' blood samples and support of the studies. This work is funded mainly by the fellowships of Shandong University for graduate students.

\section{References}

[1] J. Feely and T. E. Isles, "Screening for thyroid dysfunction in diabetics," The British Medical Journal, vol. 1, no. 6179, p. 1678, 1979.
[2] R. S. Gray, D. Q. Borsey, J. Seth, R. Herd, N. S. Brown, and B. F. Clarke, "Prevalence of subclinical thyroid failure in insulin-dependent diabetes," Journal of Clinical Endocrinology and Metabolism, vol. 50, no. 6, pp. 1034-1037, 1980.

[3] G. Gambelunghe, F. Forini, S. Laureti et al., "Increased risk for endocrine autoimmunity in Italian type 2 diabetic patients with GAD65 autoantibodies," Clinical Endocrinology, vol. 52, no. 5, pp. 565-573, 2000.

[4] S. Oh-I, H. Shimizu, T. Satoh et al., "Identification of nesfatin-1 as a satiety molecule in the hypothalamus," Nature, vol. 443, no. 7112, pp. 709-712, 2006.

[5] H. Shimizu, S. Oh-I, K. Hashimoto et al., "Peripheral administration of nesfatin-1 reduces food intake in mice: the leptinindependent mechanism," Endocrinology, vol. 150, no. 2, pp. 662-671, 2009.

[6] Y. Su, J. Zhang, Y. Tang, F. Bi, and J.-N. Liu, "The novel function of nesfatin-1: anti-hyperglycemia," Biochemical and Biophysical Research Communications, vol. 391, no. 1, pp. 1039-1042, 2010.

[7] M. Nakata, K. Manaka, S. Yamamoto, M. Mori, and T. Yada, "Nesfatin-1 enhances glucose-induced insulin secretion by promoting $\mathrm{Ca}^{2+}$ influx through L-type channels in mouse islet $\beta$ cells," Endocrine Journal, vol. 58, no. 4, pp. 305-313, 2011.

[8] G. C. Brailoiu, S. L. Dun, E. Brailoiu et al., "Nesfatin-1: distribution and interaction with a $G$ protein-coupled receptor in the rat brain," Endocrinology, vol. 148, no. 10, pp. 5088-5094, 2007.

[9] Z. Orban, S. R. Bornstein, and G. P. Chrousos, "The interaction between leptin and the hypothalamic-pituitary-thyroid axis," Hormone and Metabolic Research, vol. 30, no. 5, pp. 231-235, 1998.

[10] C. J. Price, T. D. Hoyda, W. K. Samson, and A. V. Ferguson, "Nesfatin-1 influences the excitability of paraventricular nucleus neurones," Journal of Neuroendocrinology, vol. 20, no. 2, pp. 245-250, 2008.

[11] B. Sawicka, A. Bossowski, M. Szalecki et al., "Relationship between metabolic parameters and thyroid hormones and the level of gastric peptides in children with autoimmune thyroid diseases," Journal of Pediatric Endocrinology and Metabolism, vol. 23, no. 4, pp. 345-354, 2010.

[12] Y. Taché and A. Stengel, "Regulation of food intake: the gastric X/A-like endocrine cell in the spotlight," Current Gastroenterology Reports, vol. 11, no. 6, pp. 448-454, 2009.

[13] A.-R. M. Radaideh, M. K. Nusier, F. L. Amari et al., “Thyroid dysfunction in patients with type 2 diabetes mellitus in Jordan," Saudi Medical Journal, vol. 25, no. 8, pp. 1046-1050, 2004.

[14] J. Sowiński, L. Czupryniak, A. Milewicz et al., "Recommendations of the Polish Society of Endocrinology and Polish Diabetes Association for the management of thyroid dysfunction in type 1 and type 2 diabetes," Endokrynologia Polska, vol. 64, no. 1, pp. 73-77, 2013.

[15] L. H. Duntas, J. Orgiazzi, and G. Brabant, "The interface between thyroid and diabetes mellitus," Clinical Endocrinology, vol. 75, no. 1, pp. 1-9, 2011.

[16] L. Varela, N. Martínez-Sánchez, R. Gallego et al., "Hypothalamic mTOR pathway mediates thyroid hormone-induced hyperphagia in hyperthyroidism," The Journal of Pathology, vol. 227, no. 2, pp. 209-222, 2012.

[17] N. Musi, M. F. Hirshman, J. Nygren et al., "Metformin increases AMP-activated protein-kinase activity in skeletal muscle of subjects with type 2 diabetes," Diabetes, vol. 51, no. 7, pp. 20742081, 2002. 
[18] X. Wu, H. Motoshima, K. Mahadev, T. J. Stalker, R. Scalia, and B. J. Goldstein, "Involvement of AMP-activated protein kinase in glucose uptake stimulated by the globular domain of adiponectin in primary rat adipocytes," Diabetes, vol. 52 , no. 6 , pp. 1355-1363, 2003.

[19] R. Gonzalez, B. K. Reingold, X. Gao, M. P. Gaidhu, R. G. Tsushima, and S. Unniappan, "Nesfatin-1 exerts a direct, glucose-dependent insulinotropic action on mouse islet $\beta$ - and MIN6 cells," Journal of Endocrinology, vol. 208, no. 3, pp. R9R16, 2011.

[20] M. Riva, M. D. Nitert, U. Voss et al., "Nesfatin-1 stimulates glucagon and insulin secretion and beta cell NUCB2 is reduced in human type 2 diabetic subjects," Cell and Tissue Research, vol. 346, no. 3, pp. 393-405, 2011.

[21] M. Yang, Z. Zhang, C. Wang et al., "Nesfatin-1 action in the brain increases insulin sensitivity through Akt/AMPK/TORC2 pathway in diet-induced insulin resistance," Diabetes, vol. 61, no. 8, pp. 1959-1968, 2012.

[22] D. Kohno, M. Nakata, Y. Maejima et al., "Nesfatin-1 neurons in paraventricular and supraoptic nuclei of the rat hypothalamus coexpress oxytocin and vasopressin and are activated by refeeding," Endocrinology, vol. 149, no. 3, pp. 1295-1301, 2008.

[23] Q.-C. Li, H.-Y. Wang, X. Chen, H.-Z. Guan, and Z.-Y. Jiang, "Fasting plasma levels of nesfatin- 1 in patients with type 1 and type 2 diabetes mellitus and the nutrient-related fluctuation of nesfatin-1 level in normal humans," Regulatory Peptides, vol. 159, no. 1-3, pp. 72-77, 2010.

[24] Z. Zhang, L. Li, M. Yang, H. Liu, G. Boden, and G. Yang, "Increased plasma levels of nesfatin-1 in patients with newly diagnosed type 2 diabetes mellitus," Experimental and Clinical Endocrinology and Diabetes, vol. 120, no. 2, pp. 91-95, 2012. 


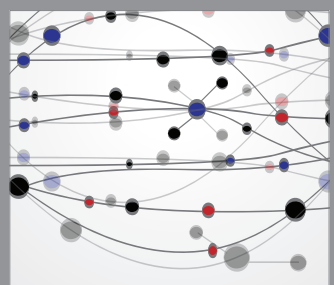

The Scientific World Journal
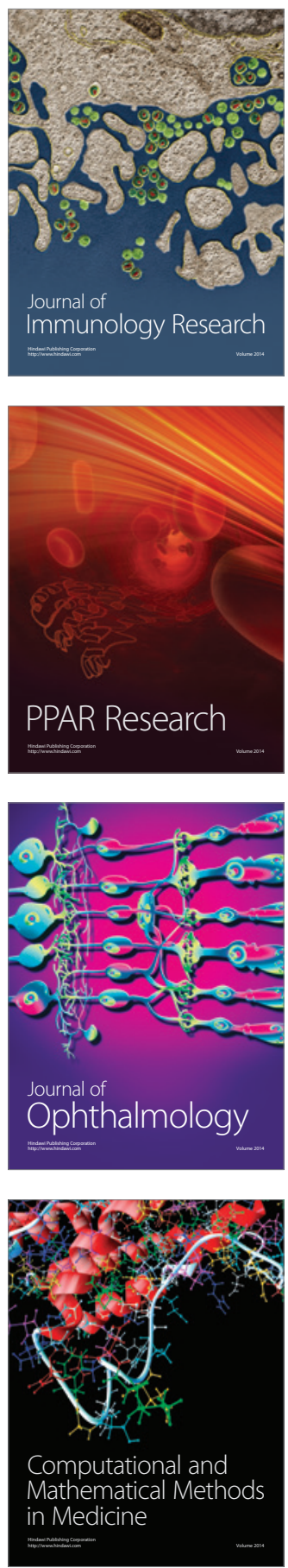

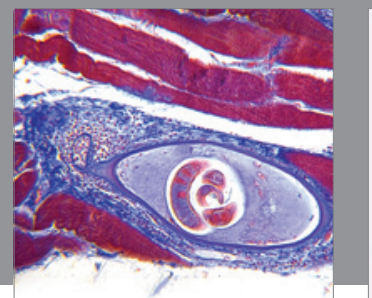

Gastroenterology

Research and Practice
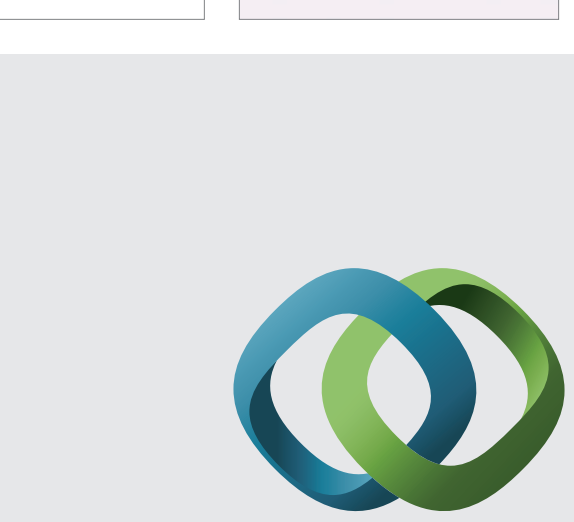

\section{Hindawi}

Submit your manuscripts at

http://www.hindawi.com
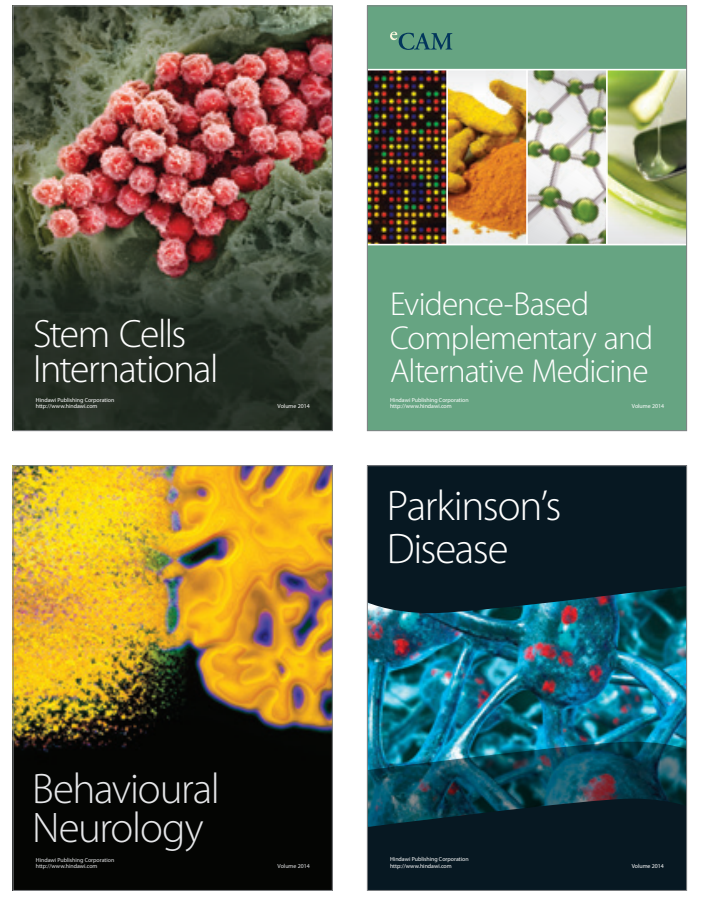
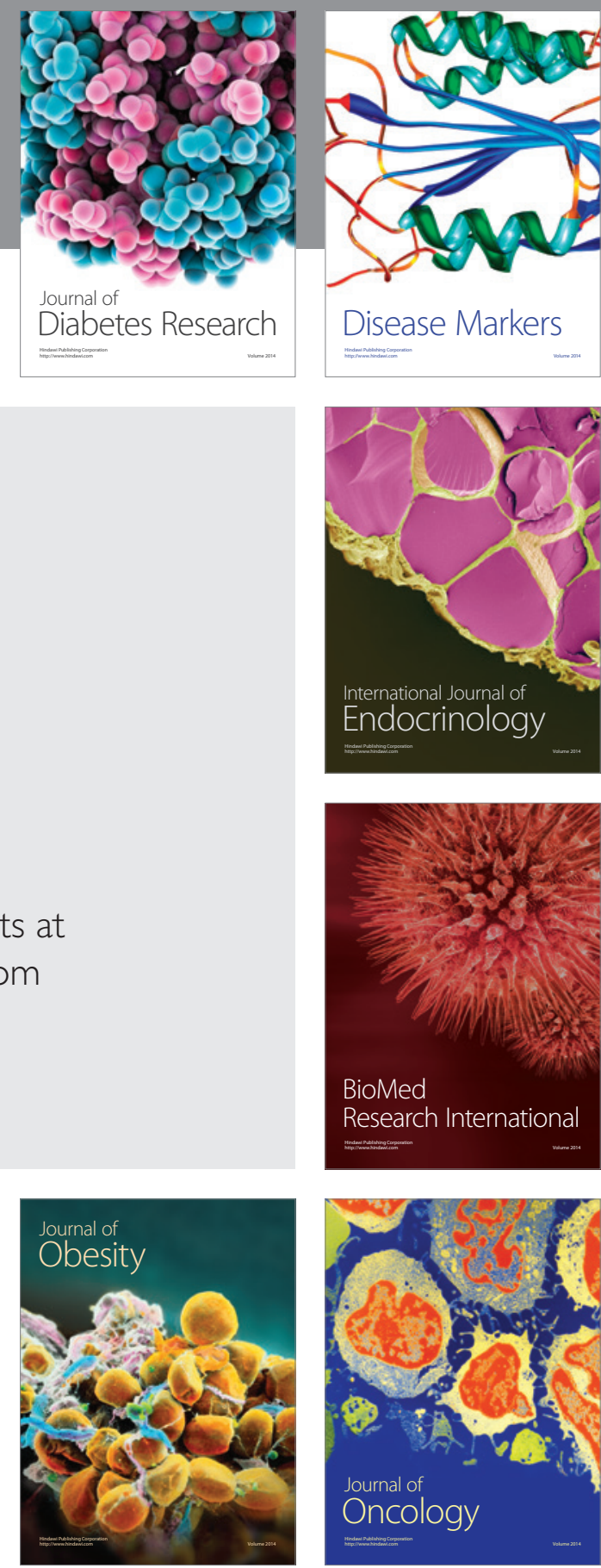

Disease Markers
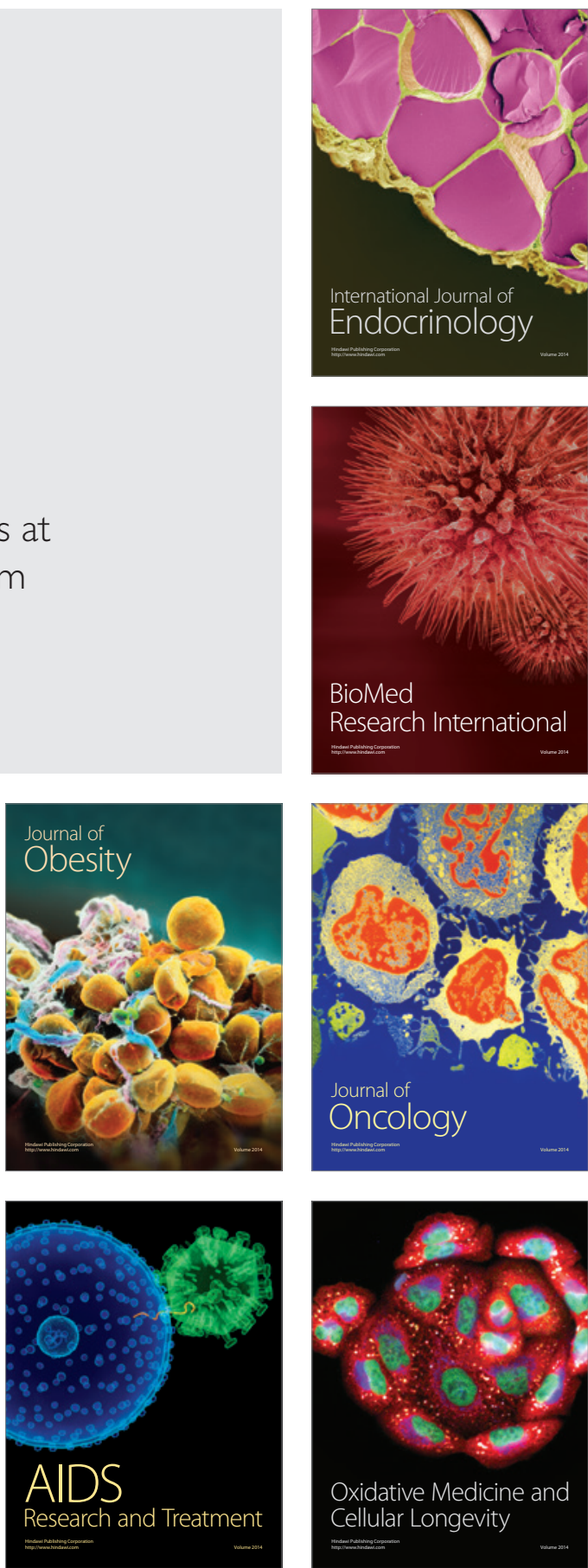